\title{
Objective Assessment of Endoscopic Surgical Skills by Analyzing Direction-Dependent Dexterity Using the Hiroshima University Endoscopic Surgical Assessment Device (HUESAD)
}

\author{
Hiroyuki Egi ${ }^{1,4}$, Masazumi Okajima ${ }^{1}$, Masanori Yoshimitsu $^{1}$, Satoshi Ikeda ${ }^{1}$, Yoshihiro Miyata ${ }^{2}$, \\ Hirokazu Masugami ${ }^{3}$, Tomohiro Kawahara ${ }^{3}$, Yuichi Kurita ${ }^{3}$, Makoto Kaneko ${ }^{3}$, and Toshimasa Asahara ${ }^{2}$ \\ ${ }^{1}$ Department of Endoscopic Surgery and Surgical Science, Graduate School of Hiroshima University, Hiroshima, Japan \\ ${ }^{2}$ Department of Surgery, Division of Frontier Medical Science, Programs for Biomedical Research, Graduate School of Biomedical Sciences, \\ Hiroshima University, Hiroshima, Japan \\ ${ }^{3}$ Department of Artificial Complex Systems Engineering, Graduate School of Engineering, Hiroshima University, Hiroshima, Japan \\ ${ }^{4}$ Department of Surgery, Hiroshima Prefectural Hospital, 1-5-54 Ujina-Kanda, Minami-ku, Hiroshima 734-8530, Japan
}

\begin{abstract}
Purpose. We evaluated our system of objectively assessing endoscopic surgical skills.

Methods. We developed the Hiroshima University Endoscopic Surgical Assessment Device (HUESAD), which records the movement of the tip of an endoscopic instrument precisely. The orbits of experienced surgeons (expert group) and those of medical students (novice group) were evaluated by measuring the deviation from the ideal course on horizontal and vertical planes. These data were integrated with the time taken to move the tip of an endoscopic instrument between a distal side pole (A) and a proximal side pole (C) (Task 1), and between a left side pole (D) and a right side pole (B) (Task 2).

Results. The integrated deviation of the expert group was significantly lower than that of the novice group on both the horizontal and vertical planes in Task $1(P=$ $0.0004, P=0.009)$ and Task $2(P<0.0001, P=0.0002)$. Thus, the spatial perception of experts was significantly better than that of novices. We also found that the direction of the scope and the movement of the endoscopic instrument were related to difficulties in spatial perception for both experts and novices. HUESAD detected and resolved these differences based on the directions of the scope and movement of the endoscopic instruments.

Conclusions. The HUESAD is a reliable system for assessing a surgeon's dexterity, based on direction and movement. It helps us to attain a higher degree of accuracy and to create an ideal setting for optimal endoscopic surgery.
\end{abstract}

Reprint requests to: H. Egi (address 4)

Received: June 11, 2006 / Accepted: April 13, 2007
Key words Assessment · Endoscopic surgery · Surgical skills · Hiroshima University Endoscopic Surgical Assessment Device (HUESAD)

\section{Introduction}

With minimally invasive surgery gaining widespread popularity, there is a need to develop a reliable system for the objective assessment of endoscopic surgical skills. The keen interest in surgeon performance, especially of endoscopic surgery, has prompted much research in this field. ${ }^{1-6}$ Endoscopic surgical skills include dexterity, knowledge, decision-making skills, communication skills, and leadership skills. Among these, dexterity is considered to be the most important; however, it is difficult to assess because it cannot be estimated by examinations. Most methods of assessing dexterity are subjective and unreliable, although in the past decade, some new assessment systems have been reported.

The objective structured assessment of technical skills (OSATS) is a system consisting of six stations where trainees perform tasks on live or bench models, and their performance is assessed by checklists and a global rating scale. ${ }^{1}$ This system has been shown to be reliable for assessing the skills of surgical residents in various simultaneous environments, ${ }^{1,7}$ but it requires many people and takes a long time.

In the next generation, motion analysis systems were developed to assess dexterity. The Imperial College surgical assessment device (ICSAD) is an electromagnetic tracking system (Isotrak II; Polhemus, Colchester, VT, USA), which consists of an electromagnetic field generator and two sensors attached to the dorsa of the surgeon's hands. This is used to assess a surgeon's dexterity by measuring the number and speed of hand 
movements, the distance traveled by the hands, and the time taken to perform a task. ${ }^{3,8-10}$ A recent report described the new advanced Dundee endoscopic psychomotor trainer (ADEPT) as being a valid and reliable method of conducting motion analysis of endoscopic surgery. ${ }^{11}$ These motion analysis systems are excellent for assessing a surgeon's dexterity.

The Hiroshima University endoscopic surgical assessment device (HUESAD) was designed to follow the movement of the tip of an endoscopic instrument precisely, based on the assumption that the orbits of the device reflect the surgeon's dexterity. The aim of this study was to evaluate the validity of HUESAD as a useful assessment system of a surgeon's dexterity and to work out how the HUESAD differs from other assessment systems.

\section{Materials and Methods}

\section{The HUESAD}

The HUESAD consists of optical scale sensors, micro encoders, an experimental table, and a monitor, which are all connected to a portable computer (Fig. 1A). This system is capable of tracking the movements of the tip of an endoscopic instrument while the operator performs tasks on the experimental table. We can measure two rotation angle parameters $(\theta, \varphi)$ and one distance parameter $(l)$ using three encoders (Fig. 1B), and record the time taken. The position of the tip of the endoscopic instrument is calculated on a portable computer, so we could record the movement of the tip of instrument precisely $(x=l \sin \varphi, y=l \cos \varphi \sin \theta, z=-l \sin \varphi \cos \theta)$ (Fig. 1C).
The HUESAD was inspected to clarify its assessment ability as follows: To minimize any error in the position measurement, we developed an error compensation method. For example, a point $\mathrm{Pm}$ was measured using the single point-based calibration, as shown in Fig. 2A; however, the measurement was inaccurate because the point $P m$ was too far away from the calibrated point $P 0$. To resolve this issue, we added another calibration point (Fig. 2B) and then estimated the tip position using these two calibrated points in the following equation:

$$
P e=s P 0+(1-s) P 1
$$

To maintain quality and accuracy, we extended this multiple point-based calibration method to the threedimensional space. The raw data of 280 examinations of this system were compared with the real information and then compensated. After compensation, the mean miscalculation was only $0.92 \pm 0.62 \mathrm{~mm}$, suggesting that the HUESAD is a high-quality system (Fig. 2C,D).

\section{Subjects}

We recruited two groups of subjects: an expert group consisting of 12 surgeons who had performed more than 100 laparoscopic procedures as the chief operator, and a novice group of 25 medical students who had performed no laparoscopic procedures. The subjects were instructed about using the assessment system. Informed consent was obtained from all participants.

\section{Experimental Design}

Task 1 involved placing the tip of the device on top of poles from $\mathrm{A}$ to $\mathrm{C}$, and then from $\mathrm{C}$ to $\mathrm{A}$ (Fig. 3A).

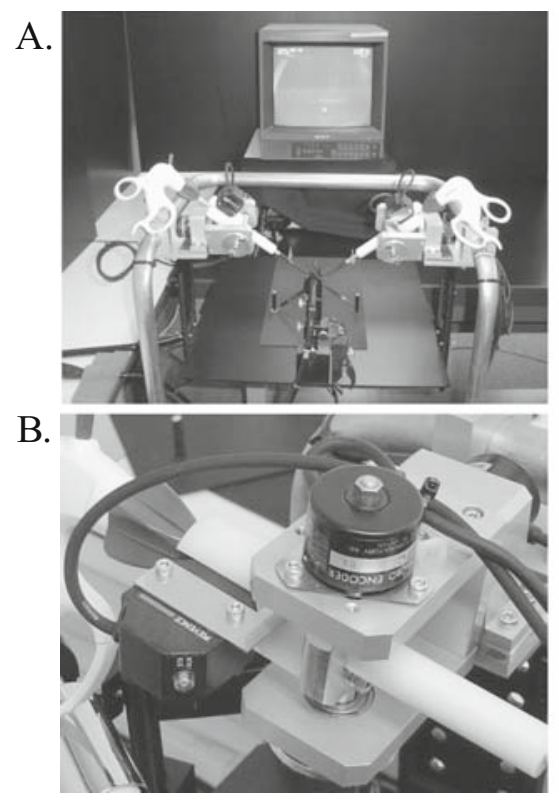

C.

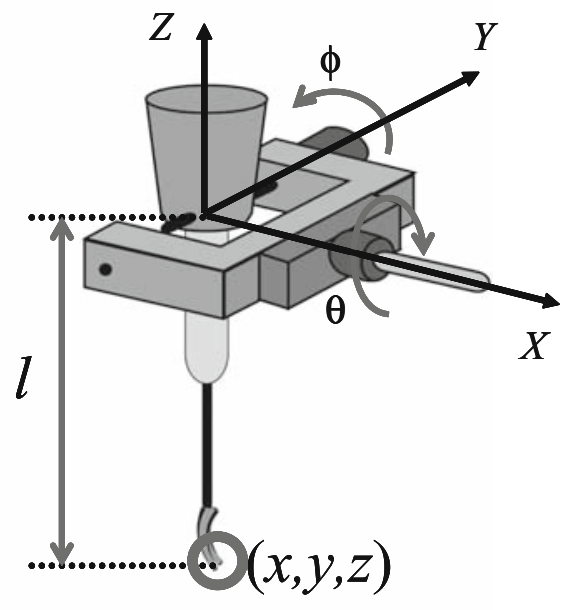

Fig. 1. A The Hiroshima University Endoscopic Surgical Assessment Device. B Micro encoders and optical scale sensors: Two rotation angle parameters $(\theta, \varphi)$ and one distance parameter $(l)$ are measured using three encoders. C The position of the tip of the endoscopic instrument is calculated and shown precisely $(x=l \sin \varphi, y=l \cos \varphi \sin \theta$, $z=-l \sin \varphi \cos \theta)$ 


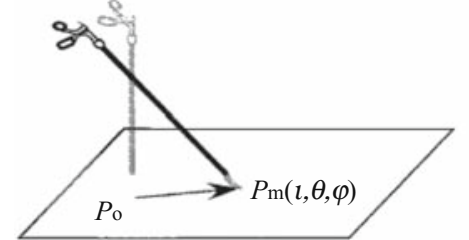

A. Single point calibration

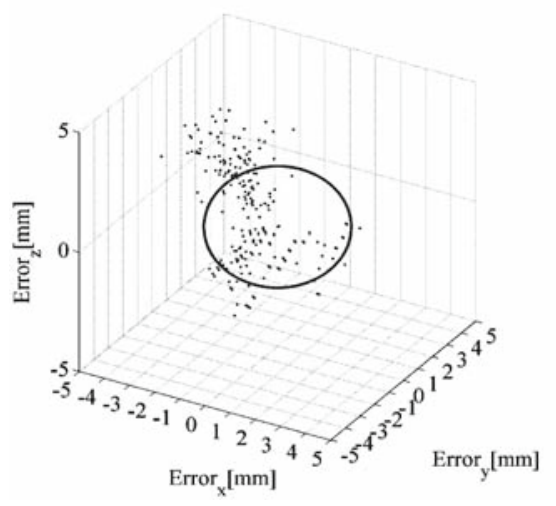

C. Before compensation

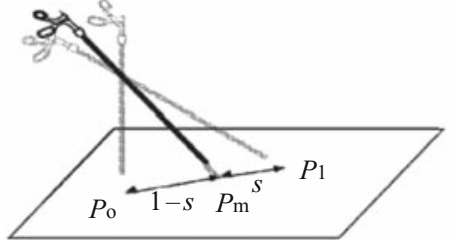

B. Multiple points calibration

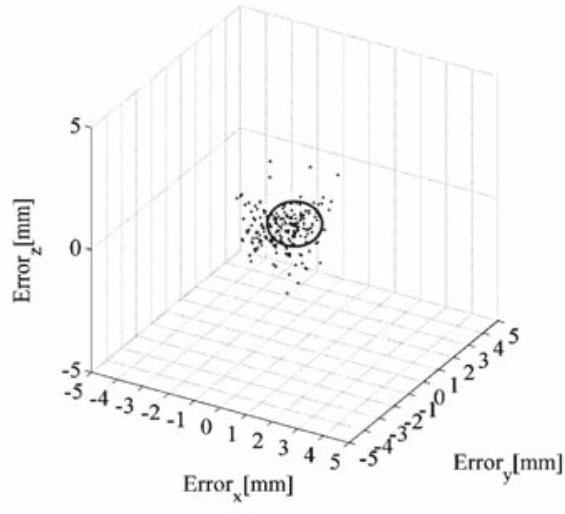

D. After compensation
Fig. 2. To decrease the error in the position measurement, we developed an error compensation method. A Single point-based calibration method. B Multiple point-based calibration method. Position errors before and after compensation: $\mathbf{C}$ before compensation; D after compensation

B.

A.

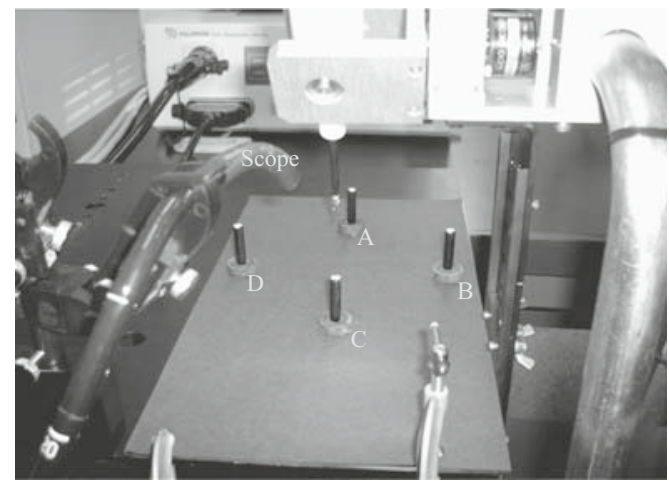

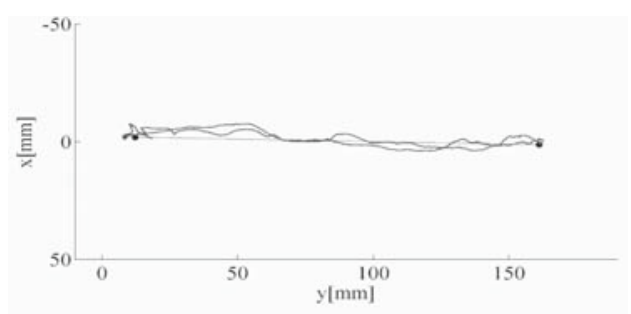

C.

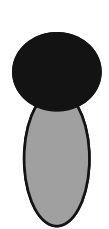

Fig. 3. A Endoscopic tasks. In Task 1, the tip of the endoscopic instrument was moved from the top of the distal pole $A$ to the proximal pole $C$ and then from $C$ to $A$. In task 2, the

Task 2 involved moving the tip of the device from the top of the left pole, D, to the top of the right pole, $\mathrm{B}$, and then moving the tip from $\mathrm{B}$ to $\mathrm{D}$. The position of the scope on the extended line from $\mathrm{A}$ to $\mathrm{C}$ is shown in Fig. 1A. Each subject repeated the tests five times using their dominant hand. The orbit of the tip of the device was accurately recorded with a portable computer.

\section{Data Analysis}

The orbits were analyzed from the top view and the lateral view (Fig. 3B,C). Deviations from the ideal courses in these two directions were measured and calculated using the following equation:

$$
\begin{aligned}
& D y=\mathrm{Z} \Delta y \mathrm{~d} x \\
& D z=\mathrm{Z} \Delta z \mathrm{~d} x
\end{aligned}
$$


Time taken for Task 1

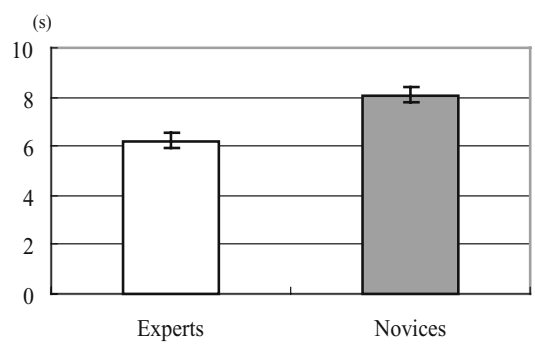

Deviation on horizontal plane of Task 1

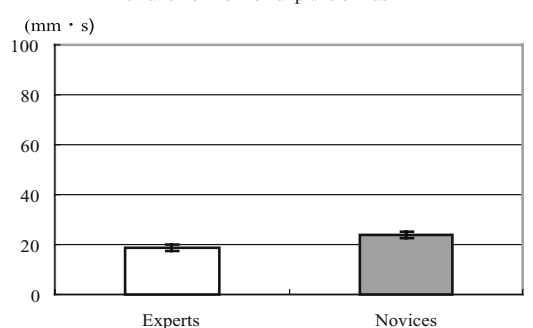

Deviation on horizontal plane of Task 2

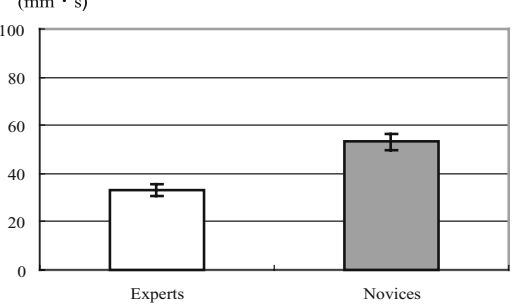

Time taken for Task 2

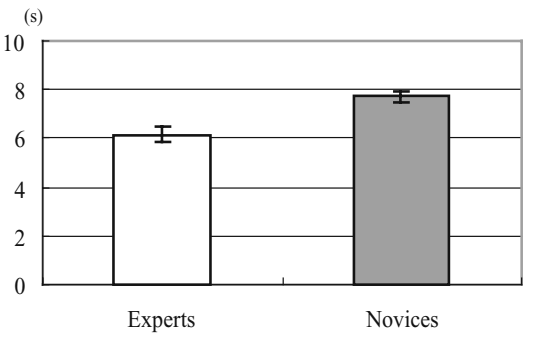

Fig. 4. The time taken by experts versus novices to perform task $1(\mathbf{A})$ and task 2 (B)

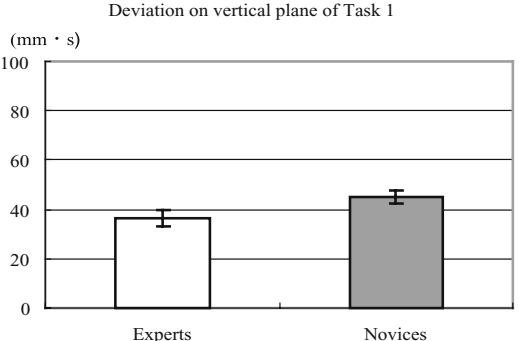

$(\mathrm{mm} \cdot \mathrm{s})$

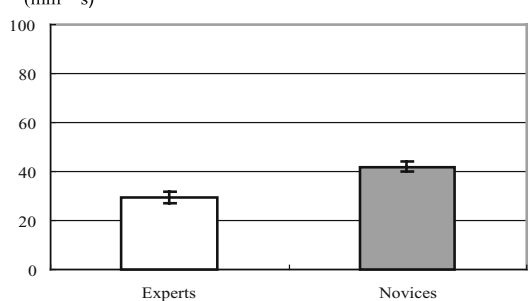

Fig. 5. Deviation from the ideal course on the horizontal and vertical planes for task 1 and task 2 (experts versus novices)
We used integrated deviation and the time taken to perform the tasks to evaluate the participants.

Statistical analysis was carried out using the statistical package Statview for Windows (Abacus, Berkeley, CA, USA). The Mann-Whitney $U$-test was used as a nonparametric test for the analysis, and the significance level was set at 0.05 .

\section{Results}

The 12 experienced surgeons in the expert group took significantly less time to perform Task 1 and Task 2 than the 25 medical students in the novice group $(P<$ $0.0001, P<0.0001$ ) (Fig. 4). The integrated deviations of both the horizontal and vertical planes for Task 1 and Task 2 were significantly lower for the expert group than for the novice group $(P=0.0004, P=0.009$ and $P<0.0001, P=0.0002$, respectively; Fig. 5). The scope position was unchanged in Task 2 . These integrated deviations of the horizontal and vertical planes for Task 1 and Task 2 were compared in the expert group. The integrated deviation of the horizontal plane for Task 1 was significantly lower than those for the others $\left({ }^{*} P<0.0001, * * P<0.0001, * * * P=0.0002\right.$; Fig. 6A). The integrated deviation of the horizontal plane for Task 1 in the novice group was also significantly lower than that for the others $\left({ }^{*} P<0.0001,{ }^{*} P<0.0001\right.$, $* * * P<0.0001$; Fig. 6B).

\section{Discussion}

There is an increasing need to establish a reliable system for the objective assessment of endoscopic surgical skills because the assessment of skills based on observations by a senior surgeon in the operating theater is subjective and unreliable. The validity and reliability of several assessment systems have been described. Szalay et al. ${ }^{12}$ described using the quality of the final product after performing six different bench model tasks to assess surgical skills. They suggested that an analysis of the final product assessment may overcome some of the problems of live ratings. The error rate is also a fundamental measurement criterion for specific skills. ${ }^{11}$ Bann et al. $^{10}$ reported that a surgical error analysis distinguishes surgeons with different levels of experience from one another, even though it is an indirect way of 


\section{A. Expert group}

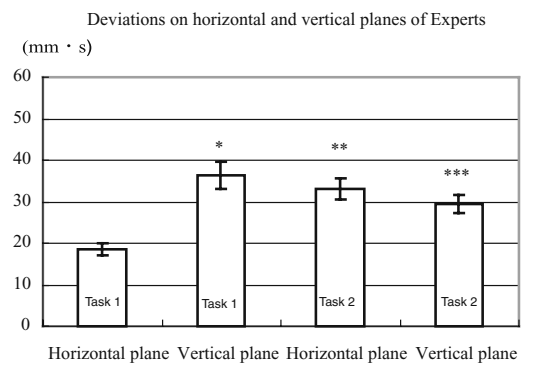

B. Novice group

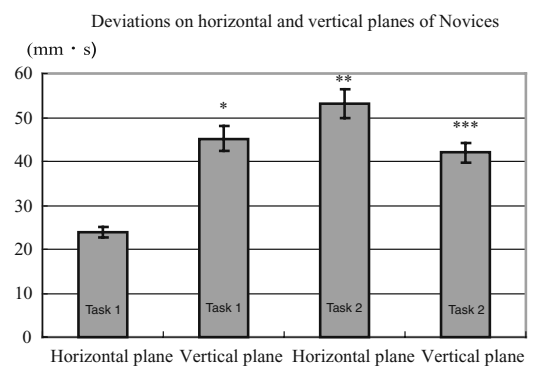

Fig. 6. A Direction-dependent dexterity in the expert group. B Direction-dependent dexterity in the novice group examining surgical skills and outcome. We believe that these systems are insufficient to assess surgical skills because skills are assessed only on the quality of the final products or error rates, whereas the process of the operation itself is not evaluated at all.

The time taken to perform a procedure has always been considered one of the most important measurements. ${ }^{13,14}$ Indeed, our results showed that the time taken by the expert group to perform Task 1 and Task 2 was significantly shorter than the time taken by the novice group (Fig. 4). In fact, the speed of the procedure has been used traditionally as an objective measure of skill. We consider the time taken to perform tasks to be one of the most important factors; yet this alone is not sufficient to assess a surgeon's dexterity accurately because a short operation does not always mean a good operation.

Minimally invasive surgery trainer-virtual reality (MIST-VR) (Mentice Medical Simulation, Gothenburg, Sweden) is a validated method of improving the skills of resident surgeons performing laparoscopic procedures. The MIST-VR system is one of the first virtual reality laparoscopic simulators. It was developed as a task trainer after close collaboration between surgeons and psychologists who performed a task analysis of a laparoscopic cholecystectomy. The outcome measures are the time needed to complete the task, the number of errors, the economy of instrument movement, and the economy of diathermy. Several studies have shown the construct validity of the MIST-VR by demonstrating its ability to consistently distinguish between experienced and inexperienced surgeons. ${ }^{15-17}$ An advantage of this system is that it records the performance of individual surgeons, but a disadvantage is that virtual reality does not allow for any force feedback. The MIST-VR system and other virtual reality systems are excellent training and assessment tools, but they all still have problems and a better system is needed.

The objective assessment of endoscopic surgical skills requires many measurements, but we believe that evaluating the process of movements in the endoscopic instrument's tip is the most important. Even if the final product is perfect or the operation time is short, an inaccurate process will be detrimental to the patient because an operation that is performed with inaccurate movements might result in injury to other organs and tissues. Thus, we devised a method that focuses on movement of the tip of endoscopic surgical device and measures the time taken to complete a task. Our method is very simple, but it provides useful information that is different from other assessment systems for evaluating endoscopic surgical dexterity.

We planned some tasks to assess a surgeon's dexterity in endoscopic surgery to establish the validity and reliability of HUESAD. The integrated deviations of the expert group were significantly lower than those of the novice group in both the horizontal and vertical planes for Task $1(P=0.0004, P=0.009)$ and Task $2(P<0.0001$, $P=0.0002$ ) (Fig. 5), indicating that the endoscopic surgical skills of experts are superior to those of novices. These results clearly show the construct validity and reliability of the HUESAD system for assessing endoscopic surgical dexterity. Interestingly, the integrated deviation on the horizontal plane for Task 1 was significantly lower than those for the others in both the expert and novice groups $(* P<0.0001, * * P<0.0001$, $* * * P=0.0002$ and $P<0.0001, P=0.0002$, respectively; Fig. 6A,B). This is an important phenomenon. One of the most difficult tasks during laparoscopic surgery is the creation of three-dimensional images from twodimensional imaging. The movement on the horizontal plane for Task 1 is able to be compensated simultaneously by the information from the two-dimensional monitor; however, other movements require the reconstruction of three-dimensional images from twodimensional information. These differences in difficulties are based on the directions of the scope and the movement of the endoscopic instrument. In other words, this phenomenon revealed that the HUESAD was able to detect the differences in difficulties and dexterity that are dependent on direction. According to a previous study, ${ }^{18}$ the pitfalls of laparoscopic surgery include an unstable camera platform, loss of the degrees of freedom, two-dimensional imaging, and poor ergonom- 
ics for the surgeons. The HUESAD enables us to solve the problem of the relationship between the direction of the scope and the endoscopic instrument, while helping the surgeon decide on the most suitable equipment for that endoscopic operation. This is the most important point since many commercially available devices are being sold throughout the world.

It has been suggested that improved training and objective assessment will reduce the degree of error. ${ }^{19}$ Another report concluded that the performance in an in vitro laparoscopic simulator correlated significantly with the performance in an in vivo animal model ${ }^{20}$ indicating that practice out of the operating room was able to improve performance in the operating room. Moreover, appropriate training requires an objective assessment system, so an objective assessment of endoscopic surgical skills is very important, not only for gaining the patient's trust, but for improving surgical treatment.

Our goal was to establish a reliable and accurate assessment system, which requires the analysis of many parameters. The findings of this study demonstrated that the HUESAD could provide a lot of information about the surgeon's visuo-spatial skills, which is one of the most important parameters for assessing endoscopic skills. Although further research is needed, the HUESAD is one of the most useful tools for assessing a surgeon's endoscopic surgical skills. The HUESAD is unique in that it can analyze new information by changing the scope position and directions of the tasks. This ability has the potential to improve the equipment used for endoscopic surgery in the operating room.

\section{References}

1. Martin JA, Regehr G, Reznick R, Macrae H, Murnahan J, Hutchison C, et al. Objective structured assessment of technical skill (OSATS) for surgical residents. Br J Surg 1997;84:273-8.

2. Reznick R, Regehr G, MacRae H, Martin J, McCulloch W. Testing technical skill via an innovative "bench station" examination. Am J Surg 1997;173:226-30.

3. Datta V, Mackay S, Chang A, Darzi A. The relationship between motion analysis and surgical technical assessments. Am J Surg 2002;184:70-3.
4. Datta V, Mackay S, Gillies D, Darzi A. Motion analysis in the assessment of surgical skill. Comput Methods Med Biomed Eng 2001;4:515-23.

5. Taffinder N, Sutton C, Fishwick RJ, McManus IC, Darzi A. Validation of virtual reality to teach and assess psychomotor skills in laparoscopic surgery: results from randomized controlled studies using the MIST-VR laparoscopic simulator. Stud Health Technol Inform 1998;50:124-30.

6. Macmillan AI, Cuschieri A. Assessment of innate ability and skills for endoscopic manipulations by the Advanced Dundee Endoscopic Psychomotor Tester: Predictive and concurrent validity. Am J Surg 1999;177:274-7.

7. Anastakis DJ, Regehr G, Reznick RK, Cusimano M, Murnaghan J. Assessment of technical skill transfer from the bench training model to the human model. Am J Surg 1999;177:167-70.

8. Darzi A, Datta V, Mackay S. The challenge for objective assessment of surgical skill. Am J Surg 2001;181:484-6.

9. Datta V, Mirren M, Mackay S, Chang A, Cheshire N, Darzi A. Relationship between skill-based model. Surgery 2002;131: 318-23.

10. Bann S, Datta V, Khan M, Darzi A. The surgical error examination is a novel method for objective technical knowledge assessment. Am J Surg 2003;185:507-11.

11. Francis NK, Hanna GB, Cuschieri A. The performance of master surgeons on the advanced Dundee endoscopic psychomotor tester: contrast validity study. Arch Surg 2002;137:841-4.

12. Szalay D, MacRae H, Regehr G, Reznick R. Using operative outcome to assess technical skill. Am J Surg 2000;180:234-7.

13. Hasson HM, Kumari NV, Eekhout J. Training simulator for developing laparoscopic skills. J Soc Laparosc Surg 2001;5: 255-65.

14. Rosser JC, Rosser LE, Savalgi RS. Objective evaluation of a laparoscopic surgical skill program for residents and senior surgeons. Arch Surg 1998;133:657-61.

15. Gallagher AG, Satava RM. Virtual reality as a metric for the assessment of laparoscopic psychomotor skills: learning curves and reliability measures. Surg Endosc 2002;16:1746-52.

16. Gallagher AG, Richie K, McClure N, McGuigan J. Objective psychomotor skills assessment of experienced, junior, and novice laparoscopists with virtual reality. Worid J Surg 2001;25: 1478-83.

17. McNatt SS, Smith CD. A computer-based laparoscopic skills assessment device differentiates experienced from novice laparoscopic surgeons. Surg Endosc 2001;15:1085-9.

18. Ballantyne GH. The pitfalls of laparoscopic surgery: challenges for robotics and telerobotic surgery. Surg Laparosc Endosc Percutan Techn 2002;12:1-5.

19. Kohn LT, Corrigan JM, Donaldson M. To err is human: building a safer health system. Washington DC: Institute of Medicine; 1999.

20. Fried GM, Derossis AM, Bothwell J, Sigman HH. Comparison of laparoscopic performance in vivo with performance measured in a laparoscopic simulator. Surg Endosc 1999;13:1077-81. 